\title{
Strain-rate effects in rheological models of inelastic response
}

\author{
V.A. Lubarda*, D.J. Benson, M.A. Meyers \\ Department of Mechanical and Aerospace Engineering, University of California, \\ San Diego; La Jolla, CA 92093-0411, USA
}

Received in final revised form 29 November 2001

\begin{abstract}
Stress-strain response under constant and variable strain-rate is studied for selected models of inelastic behavior. The derived closed-form solutions for uniaxial loading enable simple evaluation of the strain-rate effects on the material response. The effect of an abrupt change of strain-rate is also examined. Non-Newtonian viscosity which decreases with an increasing strain-rate is incorporated in the analysis. Parabolic and hyperbolic hardening are used to describe the plastic response in monotonic loading. A three-dimensional generalization of an elastic-viscoplastic model is employed to study the stress relaxation in simple shear. A combined isotropic-kinematic hardening and the concept of overstress are used in the analysis. The unloading nonlinearity of the stress-strain curve is then discussed.
\end{abstract}

(C) 2002 Published by Elsevier Science Ltd.

Keywords: A. Creep; A. Stress relaxation; B. Constitutive behavior; B. Polymeric materials; B. Viscoplastic material; C. Numerical algorithms

\section{Introduction}

The objective of this paper is an evaluation of the strain-rate effects in different rheological models of inelastic response. Two appealing models are considered based on serial and parallel combinations of elastic, viscous and plastic elements, recently used in the constitutive analysis of polymeric materials by Bardenhagen et al. (1997), and Zerilli and Armstrong (2000). A third rheological model considered is a generalized viscoelastic-viscoplastic model. The elastic response is governed by

\footnotetext{
* Corresponding author. Tel.: + 1-858-534-3169; fax: +1-858-534-5698.

E-mail address: vlubarda@ucsd.edu (V.A. Lubarda).
} 
linear or nonlinear elasticity, and the viscous response by non-Newtonian viscosity which is a decreasing function of strain-rate. The plastic element in monotonic loading is characterized by either parabolic or hyperbolic type of hardening. In the case of parabolic hardening, the flow stress is related to plastic strain by (Fig. 1)

$$
\sigma^{\mathrm{p}}=Y+\left(\hat{\sigma}^{\mathrm{p}}-Y\right) \frac{\varepsilon^{\mathrm{p}}}{\hat{\varepsilon}^{\mathrm{p}}}\left(2-\frac{\varepsilon^{\mathrm{p}}}{\hat{\varepsilon}^{\mathrm{p}}}\right),
$$

where $Y$ is the initial yield stress, and $\hat{\varepsilon}^{\mathrm{p}}$ is the plastic strain at the apex of the plastic stress-strain curve with the stress $\hat{\sigma}^{\mathrm{p}}$. The hyperbolic-type of hardening is described by

$$
\sigma^{\mathrm{p}}=\hat{Y}-\frac{\hat{Y}-Y}{1+\varepsilon^{\mathrm{p}} / \hat{\varepsilon}^{\mathrm{p}}}
$$

The limiting stress at zero saturation hardening-rate is $\hat{Y}$. The scaling strain $\hat{\varepsilon}^{\mathrm{p}}$ in Eq. (2) is conveniently specified by requiring that the initial hardening rate is equal to a prescribed value $k_{0}$ (Fig. 2). This gives $\hat{\varepsilon}^{\mathrm{p}}=(\hat{Y}-Y) / k_{0}$. In addition, an exponential type of saturation hardening is used, which is specified by

$$
\sigma^{\mathrm{p}}=Y+(\hat{Y}-Y)\left[1-\exp \left(-\frac{\varepsilon^{\mathrm{p}}}{\hat{\varepsilon}^{\mathrm{p}}}\right)\right] .
$$

In a three-dimensional generalization the plastic behavior is governed by either isotropic or anisotropic hardening. In the latter case a combined isotropic-kinematic hardening with Prager's evolution of the back stress is utilized. The equations are

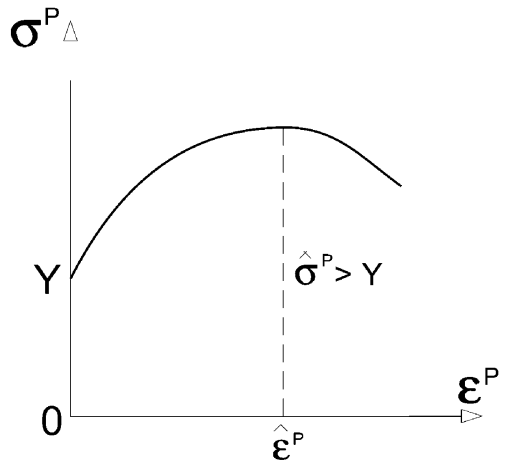

(a)



(b)

Fig. 1. Parabolic hardening plastic stress-strain curve when: (a) $\hat{\sigma}^{\mathrm{p}}>Y$, and (b) $\hat{\sigma}^{\mathrm{p}}<Y$. The initial yield stress of the material is $Y$. 
applied to study the simple shear problem of an elastic-viscoplastic material. The viscosity $\eta$ is assumed to decrease with the strain-rate $\alpha$ according to

$$
\eta(\alpha)=\eta_{\infty}+\frac{\eta_{0}-\eta_{\infty}}{\left[1+\left(\alpha / \alpha_{\mathrm{r}}\right)^{2}\right]^{n}}
$$

The viscosity parameters in the limit as $\alpha \rightarrow 0$ and $\alpha \rightarrow \infty$ are denoted by $\eta_{0}$ and $\eta_{\infty}$, respectively, and the scaling value of $\alpha$ is denoted by $\alpha_{\mathrm{r}}$. This representation of $\eta$ was proposed by Bird et al. (1977), and later used by Bardenhagen et al. (1997). Another dependence of the viscosity on the strain-rate was proposed and used by Khan and Zhang (2001).

The obtained results reproduce some of the versatile features of uniaxial response observed in polymeric materials, such as apparent softening or nonlinearities on unloading from an elastoplastic state. Numerical tests under constant strain-rate and piece-wise linear strain histories are considered, as well as under the stress relaxation and recovery conditions. This facilitates the selection of a suitable model for a particular material in the relevant range of application, and the specification of the corresponding material parameters. Related work was recently done by Khan and Liang (2000), and Khan and Zhang (2001), who modeled viscoelastic and viscoplastic deformation of BCC metals and polytetrafluoroethylene by a series connection of viscoelastic and viscoplastic elements. Both qualitative and quantitative agreement with experimental data was achieved for uniaxial static and dynamic compression, creep, and relaxation tests. Bardenhagen et al. (1997) previously modeled the stress relaxation and recovery response of an epoxy resin with a satisfactory agreement with experimental data under uniaxial loading conditions. Isothermal conditions are considered, although the temperature effects may be important, particularly in temperature sensitive polymers and at very high strain-rates.

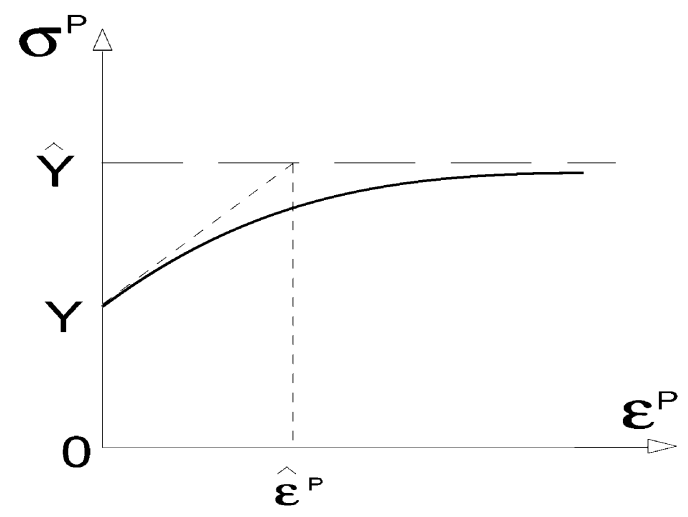

Fig. 2. Hyperbolic hardening plastic stress-strain curve. The initial yield stress is $Y$, the initial hardening rate is $k_{0}$, and the saturation hardening rate is $\hat{Y}$. 


\section{Viscoelastic-plastic model}

A series connection of elastic, viscous and plastic elements was considered by Zerilli and Armstrong (2000) as a simple one-dimensional model of polymeric response. We restrict investigations in this section to uniaxial (strain controlled) tension under constant rate of strain $\dot{\varepsilon}=\alpha=$ const. If the applied stress is below the initial yield stress $Y$, the behavior is governed by the Maxwell viscoelastic element. The stress response is

$$
\sigma=\sigma^{*}\left[1-\exp \left(-\frac{E \varepsilon}{\sigma^{*}}\right)\right], \varepsilon=-\frac{\sigma^{*}}{E} \ln \left(1-\frac{\sigma}{\sigma^{*}}\right)
$$

where $E$ is the elastic modulus, and $\eta$ the viscosity coefficient. The stress parameter $\sigma^{*}=\eta \alpha$ is explicitly introduced, since it repeatedly appears in the analysis presented in this paper. The plastic element activates at the stress level $Y$ and the corresponding strain $\varepsilon_{Y}=\varepsilon(Y)$. For $\varepsilon_{Y}$ to be finite it is required that $\sigma^{*}>Y$. Beyond the strain level $\varepsilon_{Y}$ the total rate of strain is the sum of elastic, viscous and plastic contributions, such that

$$
\dot{\varepsilon}=\frac{1}{E} \dot{\sigma}+\frac{1}{\eta} \sigma+\dot{\varepsilon}^{\mathrm{p}}
$$

In the domain of large strains the longitudinal strain $\varepsilon$ is defined as the logarithmic strain, and the stress $\sigma$ is the Cauchy stress. In the case of parabolic hardening, from Eq. (1) there follows

$$
\dot{\varepsilon}^{\mathrm{p}}= \pm \frac{\hat{\varepsilon}^{\mathrm{p}} \dot{\sigma}}{2\left[\left(\hat{\sigma}^{\mathrm{p}}-Y\right)\left(\hat{\sigma}^{\mathrm{p}}-\sigma\right)\right]^{1 / 2}}, \sigma \neq \hat{\sigma}^{\mathrm{p}}
$$

The plus sign in Eq. (7) applies in the ascending, and the minus sign in the descending portion of the stress-strain curve. The substitution of Eq. (7) into Eq. (6) yields a differential equation

$$
\left\{1 \pm \frac{E \hat{\varepsilon}^{\mathrm{p}}}{2\left[\left(\hat{\sigma}^{\mathrm{p}}-Y\right)\left(\hat{\sigma}^{\mathrm{p}}-\sigma\right)\right]^{1 / 2}}\right\} \dot{\sigma}+\frac{E}{\eta} \sigma=E \alpha .
$$

This allows a closed form solution. When $\hat{\sigma}^{\mathrm{p}}$ is the maximum achievable stress, the resulting stress-strain relationship is

$$
\varepsilon=-\frac{\sigma^{*}}{E} \ln \left(1-\frac{\sigma}{\sigma^{*}}\right)-\frac{\sigma^{*} \hat{\varepsilon}^{\mathrm{p}}}{2\left(\hat{\sigma}^{\mathrm{p}}-Y\right)^{1 / 2}\left|\hat{\sigma}^{\mathrm{p}}-\sigma^{*}\right|^{1 / 2}} \Phi^{\mathrm{m}}\left(Y, \sigma^{*}, \hat{\sigma}^{\mathrm{p}}, \sigma\right) .
$$


The function $\Phi^{\mathrm{m}}$ is defined by

$$
\Phi^{\mathrm{m}}=\ln \left|\frac{\sqrt{\hat{\sigma}^{\mathrm{p}}-\sigma}-\sqrt{\hat{\sigma}^{\mathrm{p}}-\sigma^{*}}}{\sqrt{\hat{\sigma}^{\mathrm{p}}-\sigma}-\sqrt{\hat{\sigma}^{\mathrm{p}}-\sigma^{*}}} \cdot \frac{\sqrt{\hat{\sigma}^{\mathrm{p}}-Y}+\sqrt{\hat{\sigma}^{\mathrm{p}}-\sigma^{*}}}{\sqrt{\hat{\sigma}^{\mathrm{p}}-Y}-\sqrt{\hat{\sigma}^{\mathrm{p}}-\sigma^{*}}}\right|,
$$

provided that $Y<\sigma^{*}<\hat{\sigma}^{\mathrm{p}}$ and $\sigma<\sigma^{*}$. If $\sigma^{*}>\hat{\sigma}^{\mathrm{p}}$, the function $\Phi^{\mathrm{m}}$ is

$$
\frac{1}{2} \Phi^{\mathrm{m}}= \pm \tan ^{-1} \sqrt{\frac{\hat{\sigma}^{\mathrm{p}}-\sigma}{\sigma^{*}-\hat{\sigma}^{\mathrm{p}}}}-\tan ^{-1} \sqrt{\frac{\hat{\sigma}^{\mathrm{p}}-Y}{\sigma^{*}-\hat{\sigma}^{\mathrm{p}}}} .
$$

The plus sign applies in the ascending, and the minus sign in the descending portion of the stress-strain curve. If $\sigma^{*}=\hat{\sigma}^{\mathrm{p}}$, the stress-strain response is

$$
\varepsilon=-\frac{\sigma^{*}}{E} \ln \left(1-\frac{\sigma}{\sigma^{*}}\right)+\frac{\sigma^{*} \hat{\varepsilon}^{\mathrm{p}}}{\sqrt{\sigma^{*}-Y}}\left(\frac{1}{\sqrt{\sigma^{*}-\sigma}}-\frac{1}{\sqrt{\sigma^{*}-Y}}\right) .
$$

When $\hat{\sigma}^{\mathrm{p}}$ is the minimum stress, the stress-strain relationship becomes

$$
\varepsilon=-\frac{\sigma^{*}}{E} \ln \left(1-\frac{\sigma}{\sigma^{*}}\right)+\frac{\sigma^{*} \hat{\varepsilon}^{\mathrm{p}}}{2\left(Y-\hat{\sigma}^{\mathrm{p}}\right)^{1 / 2}\left(\sigma^{*}-\hat{\sigma}^{\mathrm{p}}\right)^{1 / 2}} \Phi_{\mathrm{m}}\left(Y, \sigma^{*}, \hat{\sigma}^{\mathrm{p}}, \sigma\right) .
$$

The function $\Phi_{\mathrm{m}}$ is

$$
\Phi_{\mathrm{m}}=\ln \left(\left|\frac{\sqrt{\sigma-\hat{\sigma}^{\mathrm{p}}}-\sqrt{\sigma^{*}-\hat{\sigma}^{\mathrm{p}}}}{\sqrt{\sigma-\hat{\sigma}^{\mathrm{p}}}+\sqrt{\sigma^{*}-\hat{\sigma}^{\mathrm{p}}}}\right|^{ \pm 1} \cdot\left|\frac{\sqrt{Y-\hat{\sigma}^{\mathrm{p}}}+\sqrt{\sigma^{*}-\hat{\sigma}^{\mathrm{p}}}}{\sqrt{Y-\hat{\sigma}^{\mathrm{p}}}-\sqrt{\sigma^{*}-\hat{\sigma}^{\mathrm{p}}}}\right|\right) .
$$

The plus sign in the exponent applies to the descending, and the minus sign to the ascending portion of the stress-strain curve. The polymeric materials often show an initial descending portion of the stress-strain curve in the inelastic range, followed by an ascending (hardening) range, e.g. Boyce et al. (1988). It is noted that in the case when $\hat{\sigma}^{\mathrm{p}}$ is the minimum stress, we have $\sigma^{*}>Y>\hat{\sigma}^{\mathrm{p}}$ and $\sigma<\sigma^{*}$. The inequality $\sigma^{*} \leqslant \hat{\sigma}^{\mathrm{p}}$ does not arise because it is assumed that $\varepsilon_{Y}$ is finite, and thus $\sigma^{*}>Y$.

Numerical results are shown in Figs. 3-5. The stress-strain curves in Fig. 3 are obtained from Eqs. (9) and (10). Since in this case $\sigma<\sigma^{*}<\hat{\sigma}^{\mathrm{p}}$, the peak stress level $\hat{\sigma}^{\mathrm{p}}$ is never reached, and the response beyond the initial yield stress is always in the hardening range. Both, the current flow stress and the hardening rate increase with the increasing value of the parameter $\sigma^{*}=\eta \alpha$. The initial yield stress $Y$ is assumed to be independent of the strain-rate and equal to $100 \mathrm{MPa}$. Other parameters are $E=15 Y, \hat{\sigma}^{\mathrm{p}}=2 Y$, and $\hat{\varepsilon}^{\mathrm{p}}=0.3$. If $\sigma^{*} \leqslant Y$, the response is purely viscoelastic throughout the course of deformation, and $\sigma \rightarrow \sigma^{*}$ in the limit as $\varepsilon \rightarrow \infty$. Fig. 4 gives the stress-strain curves obtained from Eqs. (9) and (11) for the representative values of $\sigma^{*}$ that are greater than $\hat{\sigma}^{\mathrm{p}}$. Although $Y$ and $\hat{\sigma}^{\mathrm{p}}$ are both assumed to be strain-rate independent, the results indicate the features of an apparent "brittle-to- 




Fig. 3. The stress-strain curves in uniaxial tension for viscoelastic-plastic model in the case of parabolic hardening, when $\hat{\sigma}^{\mathrm{p}}>Y$ and $\sigma^{*} \leqslant \hat{\sigma}^{\mathrm{p}}$. The curves correspond to indicated values of the stress parameter $\sigma^{*}$. The material parameters are $E=15 Y, \hat{\sigma}^{\mathrm{p}}=2 Y, \hat{\varepsilon}^{\mathrm{p}}=0.3$, and $Y=100 \mathrm{MPa}$.

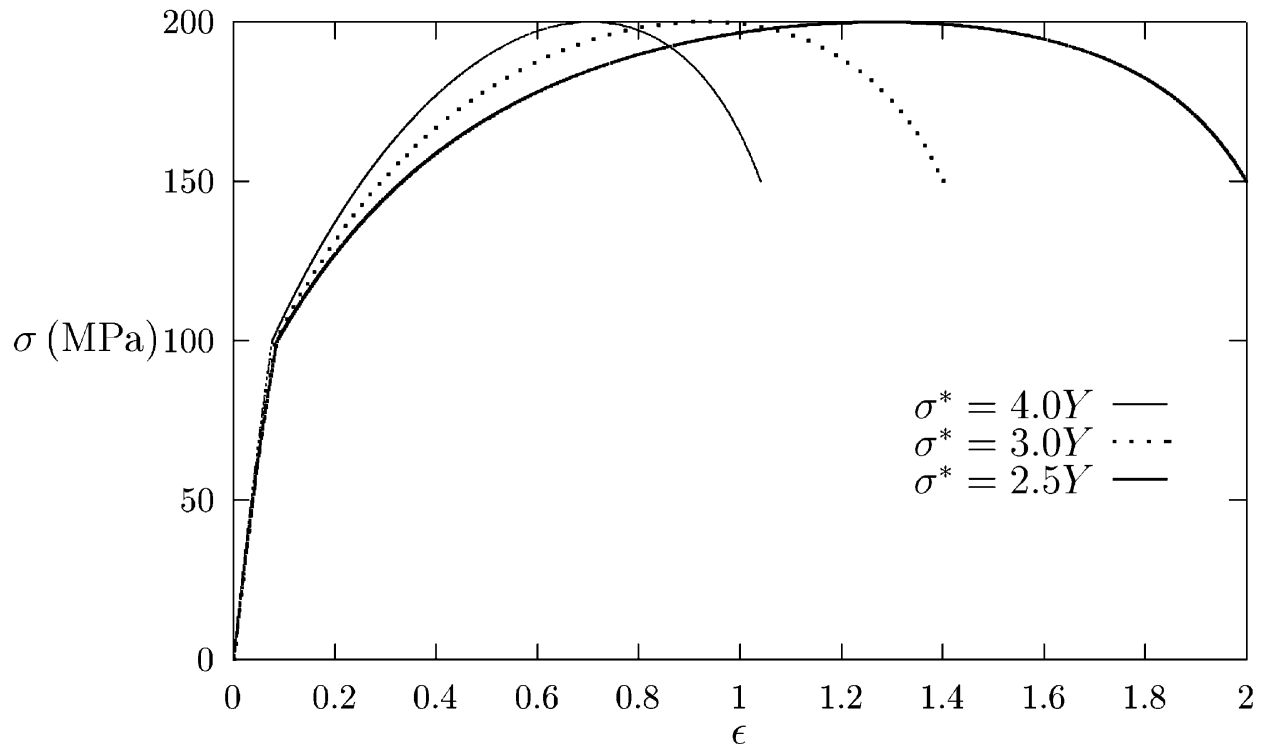

Fig. 4. The stress-strain curves for viscoelastic-plastic model in the case of parabolic hardening, when $\hat{\sigma}^{\mathrm{p}}>Y$ and $\sigma^{*} \geqslant \hat{\sigma}^{\mathrm{p}}$. The material parameters are $E=15 Y, \hat{\sigma}^{\mathrm{p}}=2 Y, \hat{\varepsilon}^{\mathrm{p}}=0.3$, and $Y=100 \mathrm{MPa}$. 
ductile" transition with a decrease of $\sigma^{*}$. The deformation beyond the peak stress levels is assumed to be uniform (without a localization). The results in Fig. 5 are obtained from Eqs. (13) and (14). Purely viscoelastic response occurs for $\sigma^{*} \leqslant Y$, while plasticity can take place only for $\sigma^{*}>Y$. The initial softening range is followed by the hardening range, the former being more extended smaller the value of the parameter $\sigma^{*}$.

The established dependence of the stress-strain response on the parameter $\sigma^{*}=$ $\eta \alpha$ can be conveniently used to examine the effects of strain rate. Suppose that the viscosity depends on strain-rate according to Eq. (4). If the values $\eta_{0}=200 \mathrm{GPa} \mathrm{s}$, $\eta_{\infty}=20 \mathrm{kPa} \mathrm{s}, \alpha_{\mathrm{r}}=0.3 \times 10^{-3} \mathrm{~s}^{-1}$, and $n=0.465$ are adopted, an increase of strain rate from 100 to $1000 \mathrm{~s}^{-1}$ results in an increase of stress from 132 to $152.5 \mathrm{MPa}$, at the strain level of one-half. The viscosity parameter at the strain-rate of $10 \mathrm{~s}^{-1}$ is equal to $12.46 \mathrm{MPa} \mathrm{s}$, at $100 \mathrm{~s}^{-1}$ it is $1.48 \mathrm{MPa} \mathrm{s}$, and at $1000 \mathrm{~s}^{-1}$ it is equal to 0.192 MPa s. The analysis can also be extended to include a pressure dependence by assuming that the viscosity depends on the superposed pressure (e.g., Argon, 1973; Zerilli and Armstrong, 2000). The effects of increasing pressure are similar to the effects of increasing strain-rate, since both elevate the flow stress and the hardening rate at a given level of strain. It should also be indicated that we assumed that the viscosity parameter depends on the total strain-rate, rather than its own (viscous) strain-rate, which significantly simplifies the analysis and enables the closed-form solutions.

In the case of hyperbolic hardening and Eq. (2), the rate of plastic strain is

$$
\dot{\varepsilon}^{\mathrm{p}}=\hat{\varepsilon}^{\mathrm{p}} \frac{\hat{Y}-Y}{(\hat{Y}-\sigma)^{2}} \dot{\sigma}
$$

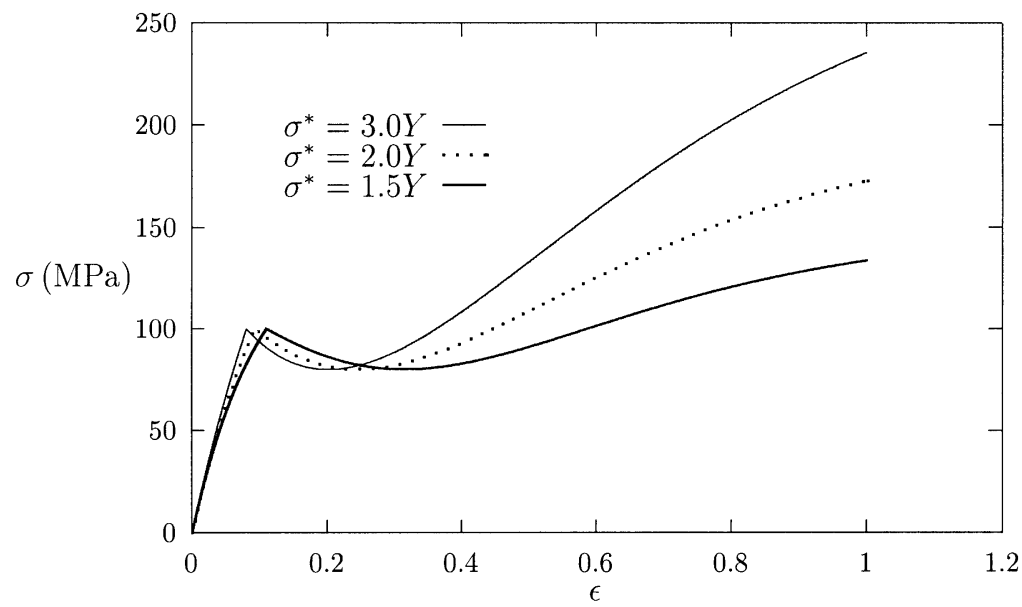

Fig. 5. The stress-strain curves for viscoelastic-plastic model in the case of parabolic hardening, when $\hat{\sigma}^{\mathrm{p}}<Y$ and $\sigma^{*} \geqslant Y$. The material parameters are $E=15 Y, \hat{\sigma}^{\mathrm{p}}=0.8 Y, \hat{\varepsilon}^{\mathrm{p}}=0.1$, and $Y=100 \mathrm{MPa}$. 
and substitution into Eq. (6) yields a differential equation

$$
\left[1+E \hat{\varepsilon}^{\mathrm{p}} \frac{\hat{Y}-Y}{(\hat{Y}-\sigma)^{2}}\right] \dot{\sigma}+\frac{E}{\eta} \sigma=E \alpha .
$$

The resulting stress-strain expression is

$$
\varepsilon=-\frac{\sigma^{*}}{E} \ln \left(1-\frac{\sigma}{\sigma^{*}}\right)-\hat{\varepsilon}^{\mathrm{p}} \frac{\hat{Y}-Y}{\hat{Y}-\sigma^{*}} \Phi\left(Y, \hat{Y}, \sigma^{*}, \sigma\right),
$$

where

$$
\Phi=\frac{\sigma^{*}}{\hat{Y}-\sigma}-\frac{\sigma^{*}}{\hat{Y}-Y}-\frac{\sigma^{*}}{\hat{Y}-\sigma^{*}} \ln \left|\frac{\sigma^{*}-Y}{\hat{Y}-Y} \cdot \frac{\hat{Y}-\sigma}{\sigma^{*}-\sigma}\right| .
$$

If $\sigma^{*}=\hat{Y}$, in place of Eq. (17), we have

$$
\varepsilon=-\frac{\sigma^{*}}{E} \ln \left(1-\frac{\sigma}{\sigma^{*}}\right)+\hat{\varepsilon}^{\mathrm{p}} \frac{\sigma^{*}(\hat{Y}-Y)}{2}\left[\frac{1}{\left(\sigma^{*}-\sigma\right)^{2}}-\frac{1}{\left(\sigma^{*}-Y\right)^{2}}\right] .
$$

Fig. 6 shows shows the stress-strain curves when $\sigma^{*}$ is equal to $1.5 Y, 2 Y$, and $3 Y$. The limiting stress value used in the calculation was $\hat{Y}=2 Y$. The other material parameters were $E=15 Y, \hat{Y}=2 Y, Y=100 \mathrm{MPa}$, and $\hat{\varepsilon}^{\mathrm{p}}=0.2$.

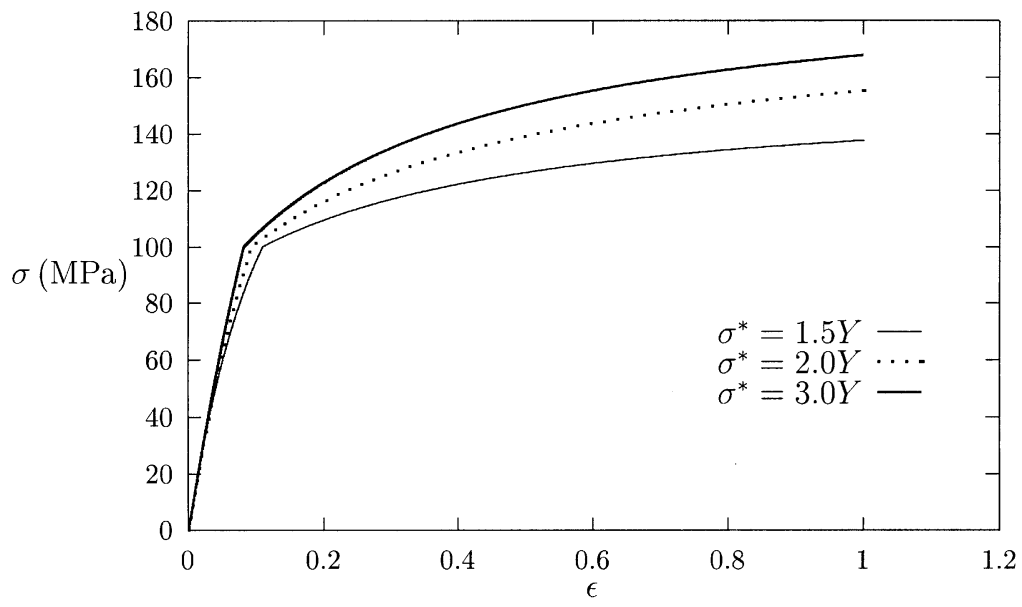

Fig. 6. The stress-strain curves for viscoelastic-plastic model in the case of hyperbolic hardening, when $\hat{Y}=2 Y$. The material parameters are $E=15 Y, \hat{\varepsilon}^{\mathrm{p}}=0.2$, and $Y=100 \mathrm{MPa}$. 
The generalizations of the simple one-dimensional model leading to Eq. (6) can be made by using the network of parallel viscoelastic elements connected in series with a plastic element (as in Zerilli and Armstrong, 2000). A nonlinear viscosity law in which the viscous part of strain rate depends nonlinearly on stress can also be used. These generalizations may be particularly important for reproducing the behavior observed in creep and relaxation tests.

\section{Viscoelastic-elastoplastic model}

A parallel connection of the viscoelastic and elastoplastic element was considered by Bardenhagen et al. (1997) as another one-dimensional model of polymeric response (Fig. 7). If $\varepsilon \leqslant Y / E$, the stress is $\sigma=E \varepsilon+\sigma^{\mathrm{v}}$, where $\sigma^{\mathrm{v}}$ is the part of stress carried by the viscoelastic element. This is governed by

$$
\dot{\varepsilon}=\frac{1}{E_{0}} \dot{\sigma}^{\mathrm{v}}+\frac{1}{\eta} \sigma^{\mathrm{v}}
$$

In the constant strain-rate test $\dot{\varepsilon}=\alpha=$ const., the integration gives

$$
\sigma^{\mathrm{v}}=\sigma^{*}\left[1-\exp \left(-\frac{E^{0} \varepsilon}{\sigma^{*}}\right)\right], \sigma^{*}=\eta \alpha
$$

If $\varepsilon \geqslant Y / E$, the total stress is

$$
\sigma=\sigma^{\mathrm{p}}+\sigma^{\mathrm{v}}=\sigma^{\mathrm{p}}+\sigma^{*}\left[1-\exp \left(-\frac{E_{0} \varepsilon}{\sigma^{*}}\right)\right]
$$

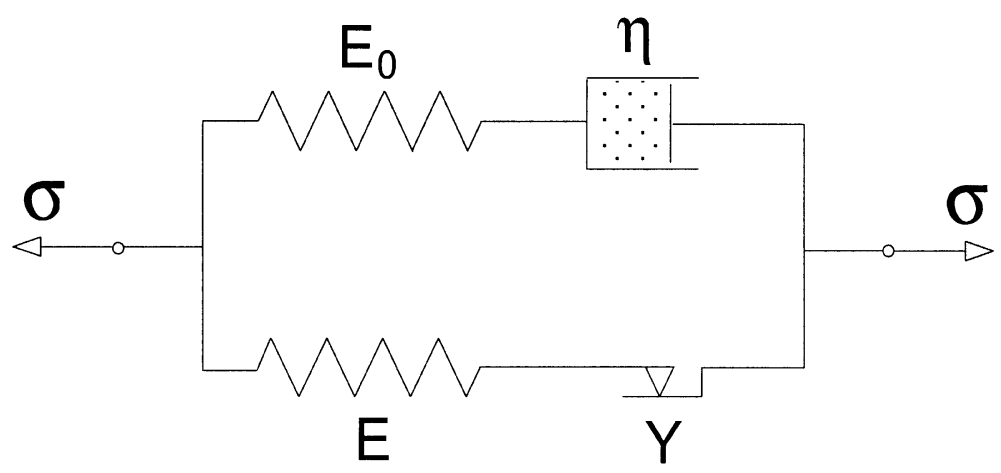

Fig. 7. Viscoelastic-elastoplastic model of uniaxial response, comprised of a parallel connection of viscoelastic and elastoplastic elements. The elastic moduli are $E$ and $E_{0}$, the viscosity coefficient is $\eta$, and the initial yield stress of the plastic element is $Y$. 
where $\sigma^{\mathrm{p}}$ is the part of stress carried by the elastoplastic element. In the case of parabolic hardening, from Eq. (1) we can write

$$
Y+\left(\hat{\sigma}^{\mathrm{p}}-Y\right) \frac{\varepsilon^{\mathrm{p}}}{\hat{\varepsilon}^{\mathrm{p}}}\left(2-\frac{\varepsilon^{\mathrm{p}}}{\hat{\varepsilon}^{\mathrm{p}}}\right)=E\left(\varepsilon-\varepsilon^{\mathrm{p}}\right) .
$$

Solving for the plastic strain $\varepsilon^{\mathrm{p}}$ in terms of the total strain $\varepsilon$ gives

$$
\frac{\varepsilon^{\mathrm{p}}}{\hat{\varepsilon}^{\mathrm{p}}}=1+\frac{1}{2\left(\hat{\sigma}^{\mathrm{p}}-Y\right)}\left\{E \hat{\varepsilon}^{\mathrm{p}}-\left[\hat{\sigma}^{2}-4\left(\hat{\sigma}^{\mathrm{p}}-Y\right)(E \varepsilon-Y)\right]^{1 / 2}\right\},
$$

where

$$
\hat{\sigma}=E \hat{\varepsilon}^{\mathrm{p}}+2\left(\hat{\sigma}^{\mathrm{p}}-Y\right) .
$$

If $\hat{\sigma}^{\mathrm{p}}>Y$, the inequality holds

$$
E \hat{\varepsilon}^{\mathrm{p}}<\left[\hat{\sigma}^{2}-4\left(\hat{\sigma}^{\mathrm{p}}-Y\right)(E \varepsilon-Y)\right]^{1 / 2}
$$

in the range $\varepsilon^{\mathrm{p}}<\hat{\varepsilon}^{\mathrm{p}}$. If $\hat{\sigma}^{\mathrm{p}}<Y$, the opposite inequality applies in (26) in the range $\varepsilon^{\mathrm{p}}<\hat{\varepsilon}^{\mathrm{p}}$. Both are ensured when

$$
\varepsilon<\hat{\varepsilon}^{\mathrm{p}}+\frac{\hat{\sigma}}{E}
$$

For strain levels greater than that given by the right-hand side of (27), a descending portion of the stress-strain response is reached in the first case, and the ascending portion in the second case. Furthermore, the collection of terms under the square-root sign in Eq. (24) must be non-negative. This is always the case when $\hat{\sigma}^{\mathrm{p}}<Y$, and is also the case when $\hat{\sigma}^{\mathrm{p}}>Y$ provided that

$$
\varepsilon<\hat{\varepsilon}^{\mathrm{p}}+\frac{\hat{\sigma}}{E}+\frac{E \hat{\varepsilon}^{\mathrm{p} 2}}{4\left(\hat{\sigma}^{\mathrm{p}}-Y\right)} .
$$

This sets the limiting value of strain, for the considered material model with a softening range, that can be reached in a test under constant rate of strain.

Returning to Eq. (24), its substitution into $\sigma^{\mathrm{p}}=E\left(\varepsilon-\varepsilon^{\mathrm{p}}\right)$ gives

$$
\sigma^{\mathrm{p}}=E \varepsilon-\frac{E \hat{\varepsilon}^{\mathrm{p}}}{2\left(\hat{\sigma}^{\mathrm{p}}-Y\right)}\left\{\hat{\sigma}-\left[\hat{\sigma}^{2}-4\left(\hat{\sigma}^{\mathrm{p}}-Y\right)(E \varepsilon-Y)\right]^{1 / 2}\right\} .
$$

The total stress follows from Eq. (22). The strain-rate dependence is present only in the expression for the viscous part of stress $\sigma^{\mathrm{v}}$. The results are shown in Fig. 8 for $\hat{\sigma}^{\mathrm{p}}=2 Y$ and $\hat{\varepsilon}^{\mathrm{p}}=0.3$. The elastic properties were $E=10 Y$ and $E_{0}=7 Y$, and $Y=100 \mathrm{MPa}$. The limiting strain value is 0.724 . Fig. 9 is obtained for $\hat{\sigma}^{\mathrm{p}}=0.5 Y$ and 
$\hat{\varepsilon}^{\mathrm{p}}=0.3$. It is characterized by an increasing rate of hardening with progression of deformation in the inelastic range.

For hyperbolic hardening and Eq. (2), we have

$$
\sigma^{\mathrm{p}}=\frac{1}{2}\left[E\left(\varepsilon+\hat{\varepsilon}^{\mathrm{p}}\right)+\hat{Y}\right]-\frac{1}{2}\left\{\left[E\left(\varepsilon-\hat{\varepsilon}^{\mathrm{p}}\right)-\hat{Y}\right]^{2}+4 E \hat{\varepsilon}^{\mathrm{p}}(E \varepsilon-Y)\right\}^{1 / 2} .
$$

The total stress $\sigma$ is again obtained from Eq. (22). The results are given in Fig. 10. The viscosity $\eta$ was determined from Eq. (4). The values $\eta_{0}=161 \mathrm{GPa} \mathrm{s}, \eta_{\infty}=16 \mathrm{kPa}$

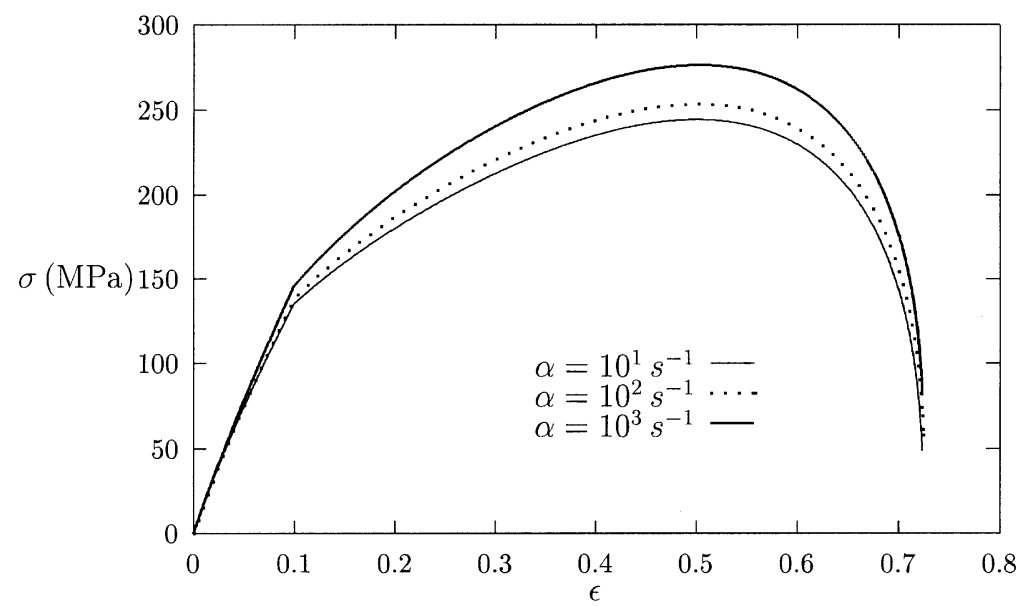

Fig. 8. The stress-strain curves in uniaxial tension for viscoelastic-elastoplastic model in the case of parabolic hardening, for the strain-rates $\alpha=10,100$, and $1000 \mathrm{~s}^{-1}$. The material parameters are $E=10 Y$, $E_{0}=7 Y, \hat{\sigma}^{\mathrm{p}}=2 Y, \hat{\varepsilon}^{\mathrm{p}}=0.3$, and $Y=100 \mathrm{MPa}$.

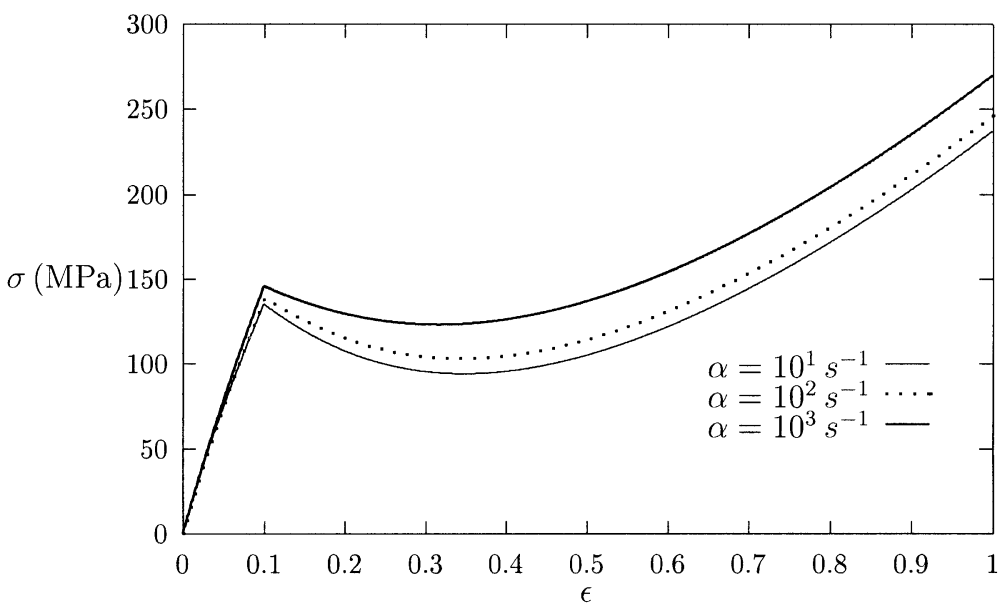

Fig. 9. The stress-strain curves for viscoelastic-elastoplastic model in the case of parabolic hardening, for the strain-rates $\alpha=10,100$, and $1000 \mathrm{~s}^{-1}$, and the material parameters $E=10 Y, E_{0}=7 Y, \hat{\sigma}^{\mathrm{p}}=0.5 Y$, $\hat{\varepsilon}^{\mathrm{p}}=0.3$, and $Y=100 \mathrm{MPa}$. 
s, $\alpha_{\mathrm{r}}=0.125 \times 10^{-3} \mathrm{~s}^{-1}$, and $n=0.465$ were used, which correspond to an epoxy resin (Bardenhagen et al., 1997). These values can be adjusted for other materials in accordance with experimental data [for example, see Khan and Zhang's (2001) proposal for a polytetrafluoroethylene data].

\section{Stress response for a piecewise-linear strain history}

Having a closed form solution for the stress-strain response under constant strainrate enables a derivation of the solution for any piecewise-linear strain history that gives rise to monotonic loading (without unloading of the plastic element). This is here demonstrated for the viscoelastic-elastoplastic material model shown in Fig. 7. Suppose that the strain level $\varepsilon_{0}$ is reached at the strain-rate $\alpha=\alpha_{0}$, and that subsequent strain segments $\varepsilon_{i}-\varepsilon_{i-1}$ are imposed at the strain rates $\alpha=\alpha_{i}$ $(i=1,2, \ldots, n)$. Beyond the strain level $\varepsilon=\varepsilon_{n}$, the strain-rate is kept at the constant value $\alpha=\alpha_{n}$. In each case the stress response is the sum of viscoelastic and plastic contributions, $\sigma=\sigma^{\mathrm{v}}+\sigma^{\mathrm{p}}$. The plastic contribution is given by Eq. (29) in the case of parabolic hardening, and by Eq. (30) in the case of hyperbolic hardening. The viscoelastic contribution can be determined from the recursive formula

$$
\sigma^{\mathrm{v}}=\sigma_{i}^{*}-\left(\sigma_{i}^{*}-\sigma_{i-1}^{\mathrm{v}}\right) \exp \left[-\frac{E_{0}\left(\varepsilon-\varepsilon_{i-1}\right)}{\sigma_{i}^{*}}\right], i=1,2,3, \ldots, n,
$$

which applies in the range $\varepsilon_{i-1} \leqslant \varepsilon \leqslant \varepsilon_{i}$. In this range the strain-rate is $\alpha=\alpha_{i}$, and the stress parameter $\sigma_{i}^{*}=\eta \alpha_{i}$. The viscoelastic stress at the strain level $\varepsilon=\varepsilon_{i-1}$ is denoted by $\sigma_{i-1}^{\mathrm{V}}$.

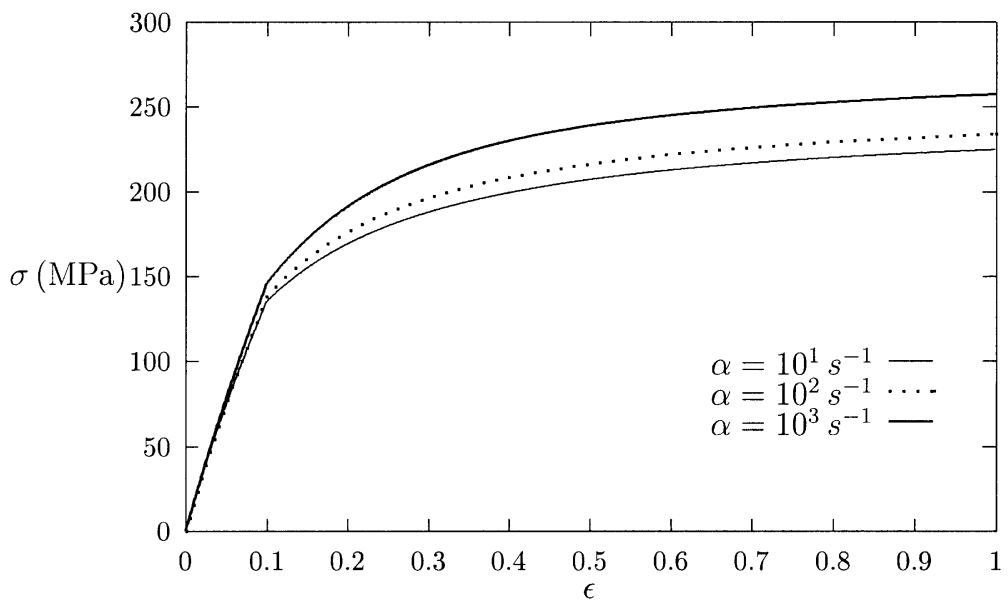

Fig. 10. The stress-strain curves in uniaxial tension for viscoelastic-elastoplastic model in the case of hyperbolic hardening described by Eq. (2), corresponding to strain-rates $\alpha=10,100$, and $1000 \mathrm{~s}^{-1}$. The material parameters are $E=10 Y, E_{0}=7 Y, \hat{Y}=2 Y, \hat{\varepsilon}^{\mathrm{p}}=0.2$, and $Y=100 \mathrm{MPa}$. 
Consider the piecewise-linear strain histories $O A_{0} A_{1} A_{2} A_{3}$ and $O A_{0}^{\prime} A_{1}^{\prime} A_{2} A_{3}$, symmetric about the path of constant strain-rate $\varepsilon=\alpha_{1} t$ (Fig. 11). The strain-rate along the segment $O A_{0}$ is $\alpha_{0}<\alpha_{1}$, and along the segment $A_{1} A_{2}$ it is $\alpha_{2}>\alpha_{1}$. The strainrate along the segment $O A_{0}^{\prime}$ is $\alpha_{0}^{\prime}=\alpha_{2}$, and along the segment $A_{1}^{\prime} A_{2}^{\prime}$ it is $\alpha_{2}^{\prime}=\alpha_{0}$. The strain-rates along the segments $A_{0} A_{1}$ and $A_{0}^{\prime} A_{1}^{\prime}$ are both equal to $\alpha_{1}$. The points $A_{0}$ and $A_{1}$ are selected so that the points $B$ and $C$ divide the segment $\overline{O A}_{2}$ symmetrically in the ratio $1: k$, i.e. the lengths $\overline{O B}=\overline{O A}_{2} /(k+1)$ and $\overline{O C}=k \overline{O A}_{2} /(k+1)$, for some $k$. Suppose that the coordinates of the point $A_{2}\left(t_{2}, \varepsilon_{2}\right)$ are $t_{2}=(k+1) \tau$ and $\varepsilon_{2}=(k+1) \alpha_{1} \tau$, where $\tau$ is a suitable time scale. It is readily found that

$$
\begin{aligned}
& \frac{t_{0}}{\tau}=\frac{1+\alpha_{1}^{2} \tau^{2}}{1+\alpha_{0} \alpha_{1} \tau^{2}}, \frac{t_{1}}{\tau}=\frac{k\left(1+\alpha_{1}^{2} \tau^{2}\right)+\alpha_{1}\left(\alpha_{1}-\alpha_{0}\right) \tau t_{0}}{1+\alpha_{1}^{2} \tau^{2}}, \\
& \varepsilon_{0}=\alpha_{0} t_{0}, \varepsilon_{1}=\alpha_{0} t_{0}+\alpha_{1}\left(t_{1}-t_{0}\right),
\end{aligned}
$$

and

$$
\begin{aligned}
& \frac{t_{0}^{\prime}}{\tau}=\frac{2 \alpha_{1} \tau \varepsilon_{0}+\left(1-\alpha_{1}^{2} \tau^{2}\right) t_{0} / \tau}{1+\alpha_{1}^{2} \tau^{2}}, \frac{t_{1}^{\prime}}{\tau}=\frac{2 \alpha_{1} \tau \varepsilon_{1}+\left(1-\alpha_{1}^{2} \tau^{2}\right) t_{1} / \tau}{1+\alpha_{1}^{2} \tau^{2}}, \\
& \varepsilon_{0}^{\prime}=\frac{2 \alpha_{1} t_{0}-\left(1-\alpha_{1}^{2} \tau^{2}\right) \varepsilon_{0}}{1+\alpha_{1}^{2} \tau^{2}}, \varepsilon_{1}^{\prime}=\frac{2 \alpha_{1} t_{1}-\left(1-\alpha_{1}^{2} \tau^{2}\right) \varepsilon_{1}}{1+\alpha_{1}^{2} \tau^{2}} .
\end{aligned}
$$

For $k=1$ the points $A_{0}$ and $A_{1}$, and $A_{0}^{\prime}$ and $A_{1}^{\prime}$ coincide, so that $\varepsilon_{0}=\varepsilon_{1}$ and $\varepsilon_{0}^{\prime}=\varepsilon_{1}^{\prime}$. Fig. 12 shows the corresponding stress for the strain path $O A_{0} A_{2} A_{3}$ in the

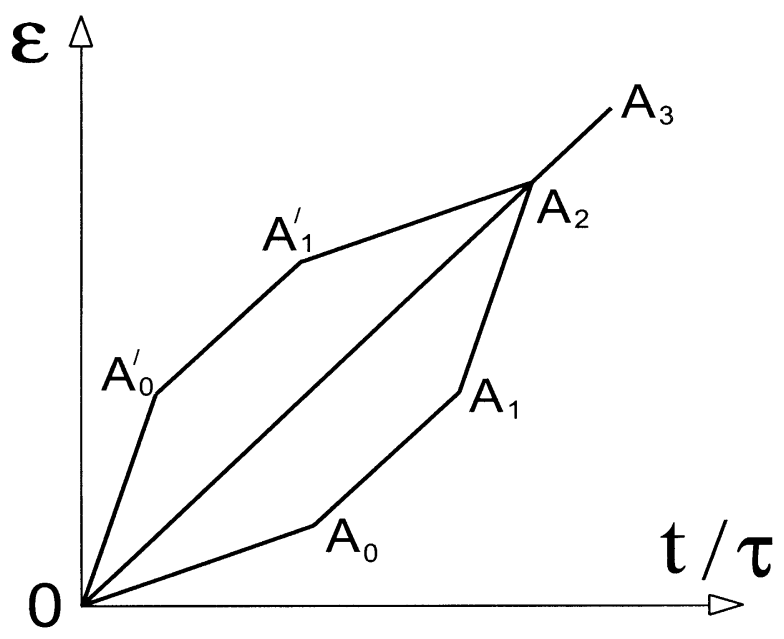

Fig. 11. Piecewise-linear strain paths $O A_{0} A_{1} A_{2} A_{3}$ and $O A_{0}^{\prime} A_{1}^{\prime} A_{2} A_{3}$, symmetric about the constant strainrate path $O A_{2} A_{3}$. The strain-rate along the segments $O A_{0}$ and $A_{1}^{\prime} A_{2}$ is $\alpha_{0}$, along the segments $A_{1} A_{2}$ and $O A_{0}^{\prime}$ it is $\alpha_{2}>\alpha_{0}$, and along $A_{0} A_{1}, A_{0}^{\prime} A_{1}^{\prime}$ and $O A_{2} A_{3}$ it is $\alpha_{1}$, where $\alpha_{0}<\alpha_{1}<\alpha_{2}$. 
case when $\alpha_{0}=50 \mathrm{~s}^{-1}$ and $\alpha_{1}=200 \mathrm{~s}^{-1}$. The strain-rate $\alpha_{2}=\alpha_{0}^{\prime}=\varepsilon_{0}^{\prime} / t_{0}^{\prime}=562 \mathrm{~s}^{-1}$ and $\alpha_{2}^{\prime}=\alpha_{0}$. The time scale is selected to be $\tau=250^{-1}$ s, so that $\varepsilon_{2}=2 \alpha_{1} \tau=1.6$. Consequently, Eqs. (32) and (33) give $t_{0}=0.5655 \times 10^{-2} \mathrm{~s}$ and $\varepsilon_{0}=0.2828$. The elastic constants are $E=10 Y$ and $E_{0}=7 Y$, where $Y=100 \mathrm{MPa}$. The hyperbolic hardening of Eq. (30) was used to describe the plastic part of the response. The viscosity parameter $\eta$ is taken to depend on the strain-rate according to Eq. (4). The stressstrain relationship corresponding to strain path $O A_{0}^{\prime} A_{2} A_{3}$ is also shown in Fig. 12. In this case from Eqs. (34) and (35) it is found that $t_{0}^{\prime}=0.2345 \times 10^{-2} \mathrm{~s}$ and $\varepsilon_{0}^{\prime}=1.3172$. The curves shown in Fig. 12 illustrate a gradual transition and the stress adjustment during monotonic loading, as the strain-rate is abruptly increased or decreased from its current value. At the strain levels sufficiently greater than $\varepsilon_{2}$, both curves approach the stress-strain curve obtained in the test at constant strain-rate $\alpha_{1}$ from the onset of deformation. Related discussion in the context of rate-dependent metal plasticity can be found in the papers by Krieg et al. (1987), and Bammann (1990).

\section{Viscoelastic-viscoplastic model}

The well-known one-dimensional rheological model depicted in Fig. 13, often used to describe viscoplastic response of metals (e.g., Malvern, 1951; Perzyna, 1966), does not allow a closed form solution for either parabolic or hyperbolic hardening plasticity. For example, in the case of parabolic hardening, a quasi-linear differential equation for the plastic strain is obtained

$$
\zeta \dot{\varepsilon}^{\mathrm{p}}+a \varepsilon^{\mathrm{p}}+b \varepsilon^{\mathrm{p} 2}=E \varepsilon-Y,
$$

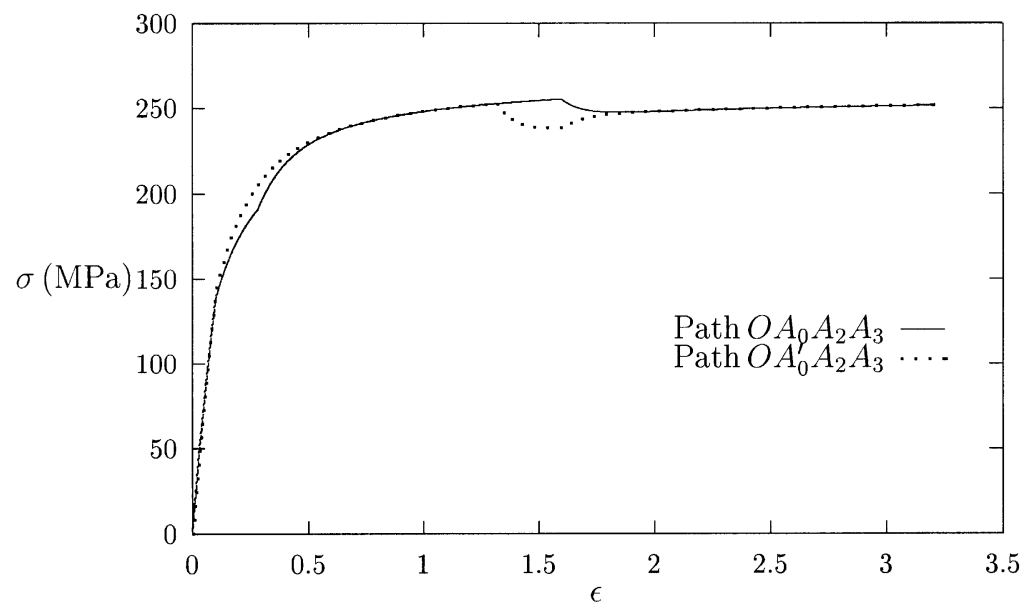

Fig. 12. The stress adjustment to an abrupt change of strain-rate during the loading program. The solid curve corresponds to the strain path $O A_{0} A_{2} A_{3}$, and the dotted curve to the strain path $O A_{0}^{\prime} A_{2} A_{3}$. 
with the initial condition $\varepsilon^{\mathrm{p}}=0$ when $\varepsilon=Y / E$. The parameters $a$ and $b$ are

$$
a=E+2 \frac{\hat{\sigma}^{\mathrm{p}}-Y}{\hat{\varepsilon}^{\mathrm{p}}}, b=-\frac{\hat{\sigma}^{\mathrm{p}}-Y}{\hat{\varepsilon}^{\mathrm{p} 2}} .
$$

Eq. (36) is a particular case of the Riccati's differential equation (e.g., Ince, 1956), which in general requires a numerical treatment. In the constant strain-rate test $\dot{\varepsilon}=\alpha$, the time-rate of plastic strain can be expressed as $\dot{\varepsilon}^{\mathrm{p}}=\alpha \mathrm{d} \varepsilon^{\mathrm{p}} / \mathrm{d} \varepsilon$, and Eq. (36) becomes

$$
\sigma_{*} \frac{\mathrm{d} \varepsilon^{\mathrm{p}}}{\mathrm{d} \varepsilon}+a \varepsilon^{\mathrm{p}}+b \varepsilon^{\mathrm{p} 2}=E \varepsilon-Y, \sigma_{*}=\zeta \alpha .
$$

Once the plastic strain is determined by numerical integration, the total stress is equal to $\sigma=E\left(\varepsilon-\varepsilon^{\mathrm{p}}\right)$. In the case of linear hardening, however, there is a closed form solution. If $\sigma^{\mathrm{p}}=Y+k_{0} \varepsilon^{\mathrm{p}}$, the plastic strain is readily found to be

$$
\varepsilon^{\mathrm{p}}=\frac{E}{E+k_{0}}\left(\varepsilon-\frac{Y}{E}\right)-\frac{E \sigma_{*}}{\left(E+k_{0}\right)^{2}}\left\{1-\exp \left[-\frac{E+k_{0}}{\sigma_{*}}\left(\varepsilon-\frac{Y}{E}\right)\right]\right\} .
$$

A combined viscoelastic-viscoplastic model is shown in Fig. 14. Two viscous elements are introduced, one with the viscosity $\eta$ and the other with the viscosity $\zeta$. Both viscosities are strain-rate dependent, but may be quite different, since they generally account for different microscopic deformation mechanisms and different time scales. Representative values of $\zeta$ for some materials can be found in Cristescu and Suliciu (1982). The total stress can be expressed as

$$
\sigma=\sigma^{\mathrm{p}}+\zeta \dot{\varepsilon}^{\mathrm{p}}
$$

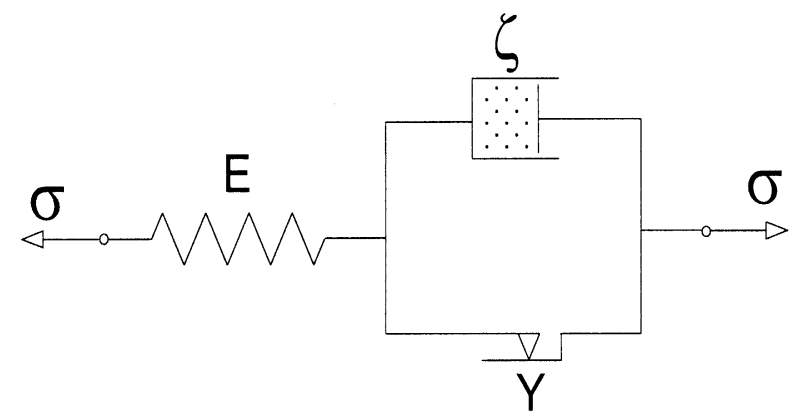

Fig. 13. Elastic--viscoplastic model of uniaxial response. Elastic element is connected in series with a viscoplastic element, comprised of a parallel connection of the viscous and plastic elements. The elastic modulus is $E$, the viscosity coefficient is $\zeta$, and the initial yield stress of plastic element is $Y$. 
where $\dot{\varepsilon}^{\mathrm{p}}$ is the rate of strain in the plastic element. If plastic hardening is linear, $\sigma^{\mathrm{p}}=Y+k_{0} \varepsilon^{\mathrm{p}}$, the total stress is

$$
\sigma=Y+k_{0} \varepsilon^{\mathrm{p}}+\zeta \dot{\varepsilon}^{\mathrm{p}},
$$

and the substitution into Eq. (6) yields

$$
\ddot{\varepsilon}^{\mathrm{p}}+\left(\frac{E}{\eta}+\frac{E+k_{0}}{\zeta}\right) \dot{\varepsilon}^{\mathrm{p}}+\frac{E k_{0}}{\eta \zeta} \varepsilon^{\mathrm{p}}=\frac{E}{\eta \zeta}(\eta \dot{\varepsilon}-Y) .
$$

In the constant strain-rate test, this becomes

$$
\ddot{\varepsilon}^{\mathrm{p}}+b \dot{\varepsilon}^{\mathrm{p}}+c \varepsilon^{\mathrm{p}}=\frac{c}{k_{0}}\left(\sigma^{*}-Y\right),
$$

where $\sigma^{*}=\eta \alpha$, and

$$
b=\frac{E}{\eta}+\frac{E+k_{0}}{\zeta}, c=\frac{E k_{0}}{\eta \zeta} .
$$

The second-order linear differential equation (43) has a general solution

$$
\varepsilon^{\mathrm{p}}=\frac{1}{k_{0}}\left(\sigma^{*}-Y\right)+C_{1} \exp \left(\frac{\rho_{1} \varepsilon}{\alpha}\right)+C_{2} \exp \left(\frac{\rho_{2} \varepsilon}{\alpha}\right),
$$

with the parameters

$$
\rho_{1,2}=\frac{1}{2}\left(-b \pm \sqrt{b^{2}-4 c}\right) .
$$



Fig. 14. Viscoelastic-viscoplastic model of uniaxial response. Viscoelastic element $(E, \eta)$ is connected in series with a viscoplastic element $(\zeta, Y)$. 
The condition $b^{2}>4 c$ is guaranteed for $k_{0}<E$. The integration constants $C_{1}$ and $C_{2}$ are determined from the conditions $\varepsilon^{\mathrm{p}}=0$ and $\dot{\varepsilon}^{\mathrm{p}}=0$, when $\sigma=Y$ and $\varepsilon=\varepsilon_{Y}$. The latter condition on the rate of plastic strain follows directly from Eq. (40). Thus,

$$
\begin{aligned}
C_{1} & =\frac{\rho_{2}}{\rho_{1}-\rho_{2}} \frac{\sigma^{*}}{k_{0}}\left(1-\frac{Y}{\sigma^{*}}\right)^{1+\rho_{1} \eta / E}, \\
C_{2} & =-\frac{\rho_{1}}{\rho_{1}-\rho_{2}} \frac{\sigma^{*}}{k_{0}}\left(1-\frac{Y}{\sigma^{*}}\right)^{1+\rho_{2} \eta / E} .
\end{aligned}
$$

This specifies the plastic strain in Eq. (45). The corresponding rate is

$$
\dot{\varepsilon}^{\mathrm{p}}=\rho_{1} C_{1} \exp \left(\frac{\rho_{1} \varepsilon}{\alpha}\right)+\rho_{2} C_{2} \exp \left(\frac{\rho_{2} \varepsilon}{\alpha}\right)
$$

Substituting Eqs. (45) and (49) into Eq. (41) gives the expression for the total stress

$$
\sigma=\sigma^{*}+\left(k_{0}+\rho_{1} \zeta\right) C_{1} \exp \left(\frac{\rho_{1} \varepsilon}{\alpha}\right)+\left(k_{0}+\rho_{2} \zeta\right) C_{2} \exp \left(\frac{\rho_{2} \varepsilon}{\alpha}\right)
$$

The results are shown in Fig. 15. Three strain-rates $\alpha=10,100$ and $1000 \mathrm{~s}^{-1}$ are imposed. The material parameters were $E=10 Y, k_{0}=2 Y$, and $Y=100 \mathrm{MPa}$. The viscosity parameter $\zeta$ was calculated from Eq. (4) and the data listed in Section 3, while $\eta$ was assumed to be 5 times greater than $\zeta$. The addition of the viscosity $\eta$ provides a nonlinear response on initial loading and viscoelastic unloading from an elastoplastic state. Note that in the relaxation test $(\dot{\varepsilon}=0$ beyond some inelastic state of deformation) the stress gradually relaxes to zero value, while for the model in Fig. 13 the stress relaxation stops when $\sigma=\sigma^{\mathrm{p}}$ (current yield stress of plastic element).

Two particular cases of the considered model are of interest. For ideal plasticity $\left(k_{0}=0\right)$, in place of Eq. (50), we have

$$
\sigma=\frac{\eta \alpha}{1+\eta / \zeta}\left\{1+\frac{Y}{\zeta \alpha}-\exp \left[-\frac{E \varepsilon}{\eta \alpha}-\frac{E\left(\varepsilon-\varepsilon_{y}\right)}{\zeta \alpha}\right]\right\}
$$

where

$$
\varepsilon_{y}=-\frac{\eta \alpha}{E} \ln \left(1-\frac{Y}{\eta \alpha}\right) .
$$

If $\eta \rightarrow \infty$, Eq. (51) reduces to

$$
\sigma=Y+\zeta \alpha\left\{1-\exp \left[-\frac{E\left(\varepsilon-\varepsilon_{y}\right)}{\zeta \alpha}\right]\right\}, \varepsilon_{y}=\frac{Y}{E} .
$$






Fig. 15. The stress-strain curves in uniaxial tension for viscoelastic-viscoplastic model. The material parameters are $E=10 Y, k_{0}=2 Y$, and $Y=100 \mathrm{MPa}$. The viscous parameter $\zeta$ is determined from Eq. (4), and $\eta=5 \zeta$.

The last expression also follows directly from Eq. (39) by letting $k_{0} \rightarrow 0$, and by making the identification $\sigma_{*}=\zeta \alpha$. Finally, if $\zeta \rightarrow 0$, Eq. (50) is replaced with

$$
\sigma=\eta \alpha\left[1-\left(1-\frac{Y}{\eta \alpha}\right) \exp \left(-\frac{E k_{0}}{E+k_{0}} \frac{E\left(\varepsilon-\varepsilon_{y}\right)}{\eta \alpha}\right)\right]
$$

which is the stress response corresponding to viscoelastic-plastic model from Section 2 , with the incorporated linear hardening plasticity. Other models of viscoplastic response can also be constructed. For example, Khan and Zhang (2001) combined in series a Zener standard solid (Maxwell viscoelastic element and elastic spring in parallel) with a viscoplastic element; see also Sobotka (1984).

\section{Stress relaxation in simple shear}

The analysis presented in previous sections was restricted to one-dimensional models of viscoplastic response. This can be variously generalized to two- and threedimensional states of stress and strain. For example, a three-dimensional generalization of the model shown in Fig. 14 gives in the case of isotropic hardening the deviatoric part of the rate of deformation as the sum of viscoelastic and viscoplastic parts, such that

$$
\mathrm{D}^{\prime}=\frac{1}{2 \mu} \stackrel{\circ}{ }^{\prime}+\frac{1}{\eta} \boldsymbol{\sigma}^{\prime}+\mathrm{D}^{\mathrm{p}}, \mathrm{D}^{\mathrm{p}}=\frac{1}{\zeta}\left\langle 1-\frac{k}{J_{2}^{1 / 2}}\right\rangle \boldsymbol{\sigma}^{\prime} .
$$




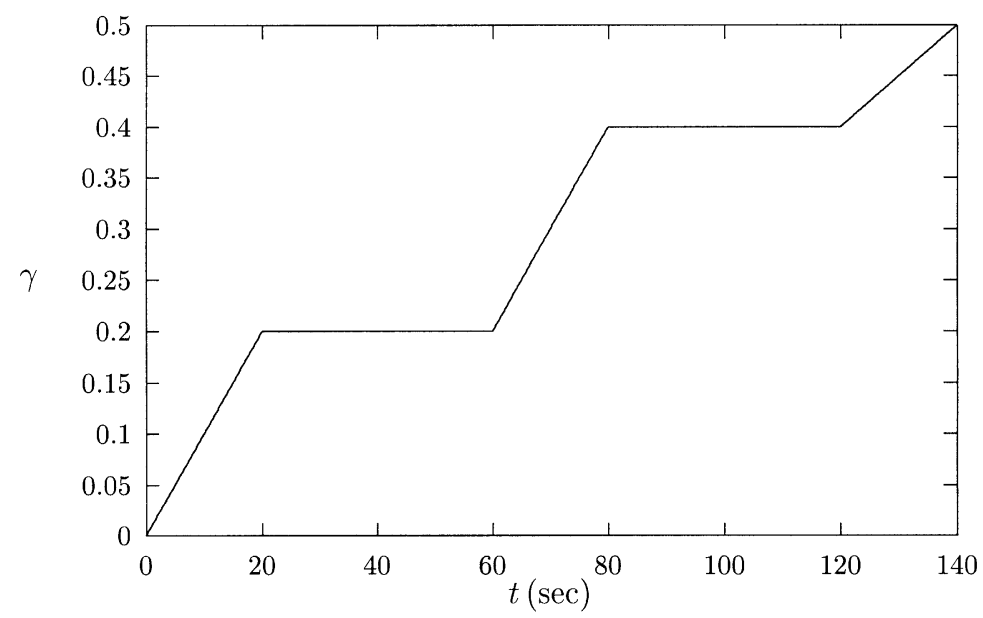

Fig. 16. The shear strain history consisting of the constant strain-rate and constant strain segments. Along the two linear segments the strain rate is $10^{-2} \mathrm{~s}^{-1}$, and along the third it is $5 \times 10^{-3} \mathrm{~s}^{-1}$.

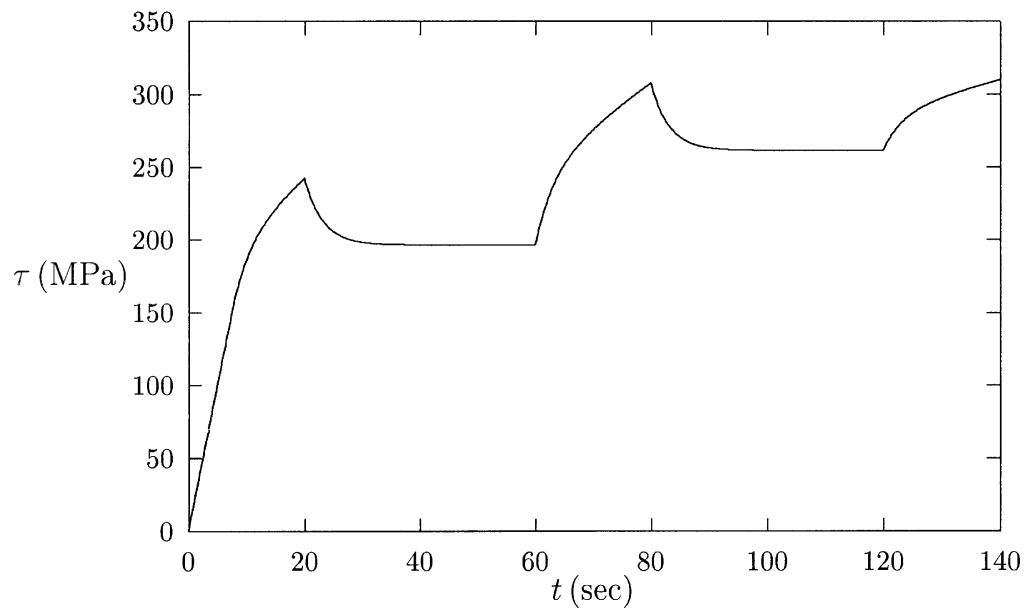

Fig. 17. The shear stress vs. time change of an elastic-viscoplastic material corresponding to piece-wise linear shear strain history from Fig. 16.

The prime designates a deviatoric part, $\mu$ is the elastic shear modulus, and superposed $\circ$ stands for the objective Jaumann derivative. The function $\langle\rightarrow$ is defined by $\langle x\rangle=x$ if $x>0$, and $\langle x\rangle=0$ if $x \leqslant 0$. The second invariant of the deviatoric Cauchy stress is $J_{2}=\left(\sigma^{\prime}: \sigma^{\prime}\right) / 2$, and

$$
k=k(\vartheta), \vartheta=\int_{0}^{t}\left(2 \mathrm{D}^{\mathrm{p}}: \mathrm{D}^{\mathrm{p}}\right)^{1 / 2} \mathrm{~d} t
$$






Fig. 18. The shear stress vs. shear strain corresponding to time histories of shear strain and shear stress from Figs. 16 and 17. The stress dips correspond to stress relaxation during the intervals of constant strain.

is a scalar which specifies the size of the current static yield surface $J_{2}=k^{2}$. The symbol : stands for the trace product. In the case of a combined isotropic-kinematic hardening with the back stress $\boldsymbol{\alpha}$, the deviatoric part of the rate of deformation is

$$
\mathrm{D}^{\prime}=\frac{1}{2 \mu} \dot{\boldsymbol{\sigma}}^{\prime}+\frac{1}{\eta} \boldsymbol{\sigma}^{\prime}+\frac{1}{\zeta}\left\langle 1-\frac{\sqrt{2} k}{\left\|\boldsymbol{\sigma}^{\prime}-\boldsymbol{\alpha}\right\|}\right\rangle\left(\boldsymbol{\sigma}^{\prime}-\boldsymbol{\alpha}\right),
$$

where $\left\|\boldsymbol{\sigma}^{\prime}-\boldsymbol{\alpha}\right\|^{2}=\left(\boldsymbol{\sigma}^{\prime}-\boldsymbol{\alpha}\right):\left(\boldsymbol{\sigma}^{\prime}-\boldsymbol{\alpha}\right)$. The Prager or Armstrong-Frederick evolution equation for the back stress can be used to specify the change of $\boldsymbol{\alpha}$. Other threedimensional models of viscoplastic response have recently been discussed by Lubarda and Benson (2002a,b), and Krempl (2001).

Consider a stress relaxation in simple shear during the intervals of constant strain shown in Fig. 16. The elastic-viscoplastic model of Eq. (57) with Prager's evolution of the back stress is used. The results of numerical integration employing a radial return method are shown in Figs. 17 and 18. The elastic shear modulus was $\mu=1$ $\mathrm{GPa}$ and the initial yield stress in quasi-static loading $70 \mathrm{MPa}$. The radius of the yield surface saturates to $150 \mathrm{MPA}$ at large values of $\vartheta$ (saturation kinematic hardening). The coefficient in the evolution equation for $\boldsymbol{\alpha}$ was equal to $250 \mathrm{MPa}$, and the viscosity parameter $\zeta$ was determined from Eq. (4) and the data listed in Section 3. The nature of stress relaxation along the plateaus of constant strain is governed by the magnitude of $\zeta$. Figs. 19 and 20 show the stress response to a loadingunloading strain history in which the shear strain of 0.2 was reached in $20 \mathrm{~s}$ at constant strain-rate, and then removed at the same magnitude of strain-rate. There is a nonlinear plastic response in the early stage of the strain removal, because the viscous stress decreases, while plastic stress increases, but less rapidly so that their sum 


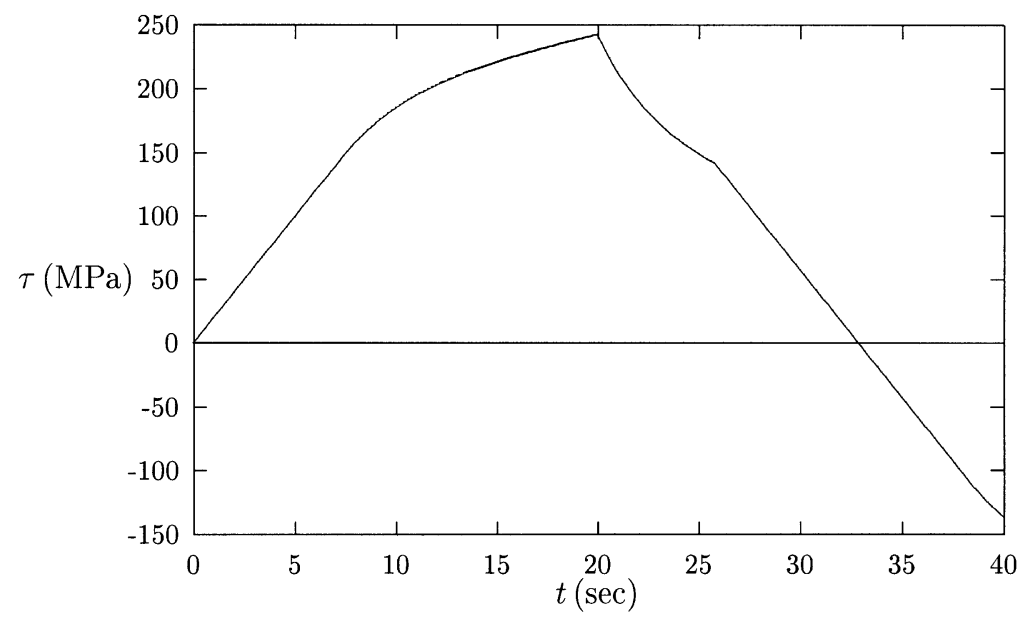

Fig. 19. The shear stress vs. time history of an elastic-viscoplastic material corresponding to a ramp-like shear strain history with a constant magnitude of the strain-rate equal to $10^{-2} \mathrm{~s}^{-1}$. The viscosity $\zeta=7.5$ GPa.

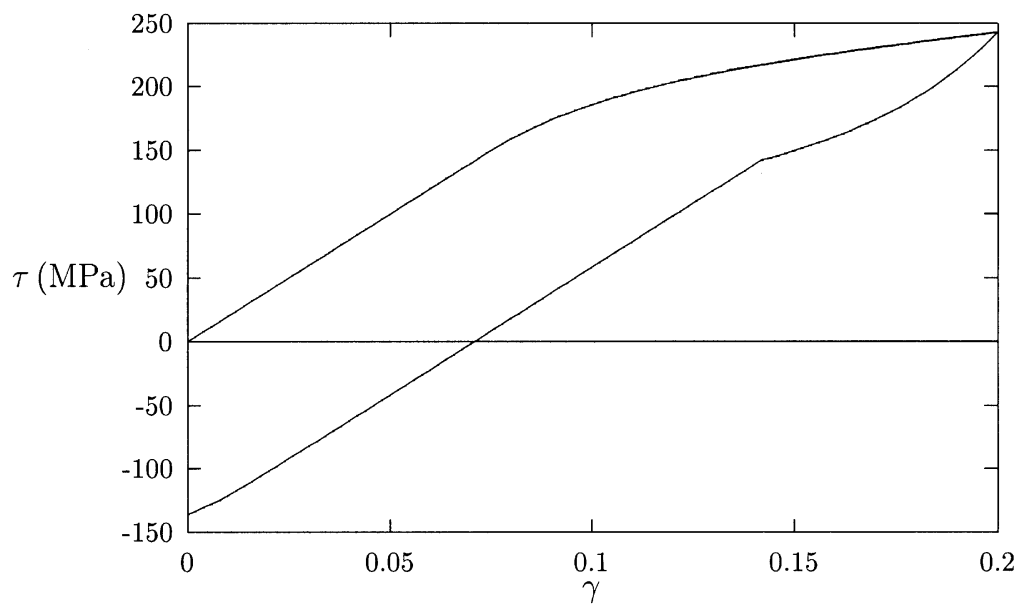

Fig. 20. The shear stress vs. shear strain corresponding to the prescribed cycle of shear strain.

continues to decrease. When the viscous stress becomes equal to zero, the viscoplastic element locks up and further unloading takes place in a purely elastic manner. The corresponding segments in Figs. 19 and 20 are linear. They can be made nonlinear by either including viscoelastic effects, or by incorporating the elastic response with a nonlinear rubber-type elasticity associated with the straightening of the polymer's long-chain molecules. The plastic flow takes place again when the compressive yield stress is reached on the reversed loading.

More general constitutive models can be constructed by combining any number of particular models, each of which may be designed to simulate a specific deformation 
mechanism. The coefficients used to link these models can be specified by an appropriate optimization procedure to achieve the best agreement with experimental data. Such procedure has already been used in modeling the anisotropic hardening, where different models are linked together in a weighted sum to reproduce the behavior in cyclic creep and relaxation tests.

\section{Acknowledgements}

Research funding provided by the Los Alamos National Laboratories is kindly acknowledged. We also thank reviewers for their comments and suggestions.

\section{References}

Argon, A.S., 1973. A theory for the law-temperature plastic deformation of glassy polymers. Philos. Mag. 28, 839-865.

Bammann, D.J., 1990. Modeling temperature and strain rate dependent large deformations of metals. Appl. Mech. Rev. 43, S312-S319.

Bardenhagen, S.G., Stout, M.G., Gray, G.T., 1997. Three-dimensional, finite deformation, viscoplastic constitutive models for polymeric materials. Mech. Mater. 25, 235-253.

Bird, R.B., Armstrong, R.C., Hassager, O., 1977. Dynamics of Polymeric Liquids, Vol. I. John Wiley and Sons, New York.

Boyce, M.C., Parks, D.M., Argon, A.S., 1988. Large inelastic deformation of glassy polymers. Part I: rate dependent constitutive model. Mech. Mater. 7, 15-33.

Cristescu, N., Suliciu, I., 1982. Viscoplasticity. Martinus Nijhoff, The Hague.

Ince, E.L., 1956. Ordinary Differential Equations. Dover, New York.

Khan, A., Liang, R., 2000. Behavior of three BCC metals during non-proportional multi-axial loadings: experiments and modeling. Int. J. Plasticity 16, 1443-1458.

Khan, A., Zhang, H., 2001. Finite deformation of a polymer: experiments and modeling. Int. J. Plasticity 17, 1167-1188.

Krempl, E., 2001. Relaxation behavior and modeling. Int. J. Plasticity 17, 1419-1436.

Krieg, R.D., Swearengen, J.C., Jones, W.B. (Eds.), 1987. Unified Constitutive Equations for Creep and Plasticity. Elsevier, London.

Lubarda, V.A., Benson, D.J. 2002. On the evolution equation for the rest stress in rate-dependent plasticity. Int. J. Plasticity, 18, 895-918.

Lubarda, V.A., Benson, D.J., 2002b. On the numerical algorithm for isotropic-kinematic hardening with the Armstrong-Frederick evolution of the back stress. Submitted for publication.

Malvern, L.E., 1951. The propagation of longitudinal waves of plastic deformation in a bar of material exhibiting a strain-rate effect. J. Appl. Mech. 18, 203-208.

Perzyna, P., 1966. Fundamental problems in viscoplasticity. Adv. Appl. Mech. 9, 243-377.

Sobotka, Z., 1984. Rheology of Materials and Engineering Structures. Elsevier, Amsterdam.

Zerilli, F.J., Armstrong, R.W., 2000. Thermal activation based constitutive equations for polymers. J. Phys. IV 10, Pr 9-3-Pr 9-8. 